$240 \mid 2012$

Varia

\title{
Paysages de Claude Gellée
}

\section{R. Stanley Johnson}

\section{OpenEdition}

\section{Journals}

Édition électronique

URL : http://journals.openedition.org/estampe/980

DOI : 10.4000/estampe.980

ISSN : 2680-4999

\section{Éditeur}

Comité national de l'estampe

\section{Édition imprimée}

Date de publication : 1 septembre 2012

Pagination : 60-63

ISSN : 0029-4888

\section{Référence électronique}

R. Stanley Johnson, «Paysages de Claude Gellée », Nouvelles de l'estampe [En ligne], 240 | 2012, mis en ligne le 15 octobre 2019, consulté le 24 septembre 2020. URL : http://journals.openedition.org/ estampe/980 ; DOI : https://doi.org/10.4000/estampe.980

\section{cc) (i)}

La revue Nouvelles de l'estampe est mise à disposition selon les termes de la Creative Commons Attribution 4.0 International License. 


\section{PAYSAGES DE CLAUDE GELLÉE}

Martin Sonnabend et Jon Whiteley (dir.), Claude Lorrain: The Enchanted Landscape, catalogue des expositions Ashmolean Museum, Oxford - Städel Museum, Francfort, Lund Humphries Publishers Ltd, 200 pages, ISBN 978-1848220928.

\section{R. Stanley Johnson}

Une exposition majeure sur Claude Gellée Le Lorrain (1600-1682) a eu lieu au Ashmolean Museum à Oxford du 6 octobre 2011 au 8 janvier 2012 et ensuite au Städel Museum à Francfort du 3 février jusqu’au 6 mai 2012. L'exposition montre un ensemble de quinze tableaux, soixante dessins et cinquante-six gravures. Les œuvres, aussi bien que le catalogue de cette exposition, ont été réunies par Jon Whiteley du Ashmolean Museum et Martin Sonnabend du Städel Museum, avec la participation de Christian Rümelin du musée d'art et d'histoire de Genève.

Il y a des tableaux magnifiques dans cette exposition. Dans la préface du catalogue, les directeurs des deux musées, Christopher Brown et Max Hollein, prennent note de deux chefs-d'œuvre de Claude, exposés ensemble pour la première fois : Ascagne tirant sur le cerf de Sylvia (Ashmolean) et Noli me tangere (Städel Museum).

Les soixante dessins de Claude choisis pour cette exposition sont tous exceptionnellement beaux. Il faudrait contempler et jouir de chacun séparément. Les textes du catalogue démontrent bien qu'il n'y a pas de paradigme parmi ces dessins de Claude. Il y a des dessins préparatoires pour et d'après des tableaux, des dessins préparatoires pour d'autres dessins ou pour des gravures, et puis des dessins indépendants, quelquesuns très esquissés, d'autres très complets. Comme dessin très complet, il y a par exemple Port avec la reine de Sheba, 1648 (Kupferstichkabinett, Berlin); comme dessin très esquissé, il y a par exemple La Vue du jardin d'arbuste, avec mur à droite, 1640 (Ashmolean). Les sujets de Claude varient : il y a des paysages pastoraux, des ports, des figures, des animaux. Ses sujets favoris semblent avoir comme source principale ses grandes promenades dans la campagna de Rome. Ces dessins de la campagna, comme indiqué par Whiteley, ont été commencés dans « l'air frais ». Après avoir esquissé les plantes, les arbres et les effets de soleil, Claude, à l'atelier, a terminé ces études sous forme de tableaux, gravures ou autres dessins.

Dans la préface de leur catalogue, les directeurs des deux musées constatent qu'audelà de tant de merveilleuses découvertes, le but particulier de cette exposition serait de « réviser un certain nombre d'idées préconçues concernant l'art de Claude. Surtout les gravures, qui dans les descriptions de son œuvre sont normalement considérées seulement comme des "after-thoughts", occupent une place majeure dans cette exposition. »

La partie la plus intrigante de cette exposition en effet concerne les gravures. Depuis plus d'un siècle, on ajoute toujours des informations et découvertes au sujet des gravures de Claude. Il y avait des catalogues raisonnés par Robert-Dumesnil (1871), Edouard Meaume (1871), Georges Duplessis (1875) et André Blum (1923). Avec cent soixante-dix-huit œuvres réunies et un catalogue descriptif par H. Diane Russell, 


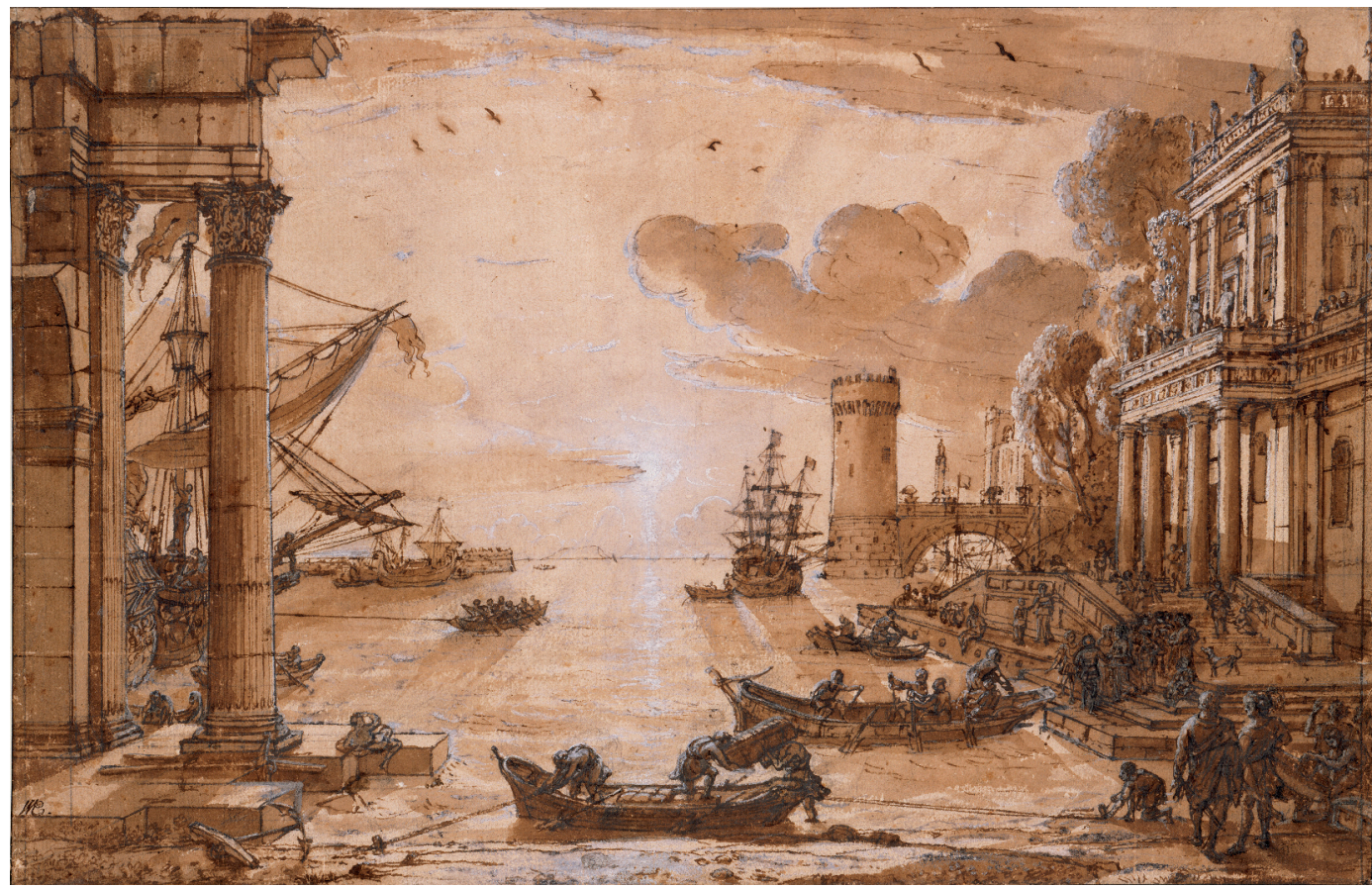

Cat. Nr. 64 Port avec la Reine de Shéba (encre, lavis avec du blanc), 1648. Kupferstichkabinett, Staatliche Museen, Berlin.

il y avait l'exposition à la National Gallery of Art à Washington DC et aux Galeries nationales du Grand Palais à Paris (1982-1983). Concernant les gravures de Claude, Russell présente maintes « corrections » qui ont trait aux catalogues du passé. Ensuite, avec encore des corrections, il y a Lino Mannocci Claude Lorrain, opera graphica (1991). Les descriptions des gravures du catalogue actuel sont fondées sur le catalogue de Mannocci, mais elles ajoutent aussi beaucoup d'informations nouvelles concernant les états, les techniques, les filigranes, et les sources des gravures de Claude.

En regardant les cinquante-six épreuves de gravures de Claude dans l'ensemble de cette exposition, il est intéressant de comparer, par exemple, la gravure La Tempeste de 1630 (premier de sept états, Ashmolean) avec un dessin de la même date et du même sujet (British Museum) : le dessin représente seulement un point de départ avant que Claude n'ait développé la gravure. Il est aussi fascinant de se rendre compte que la gravure Paysage avec des brigands, 1633 (premier état, musée du Petit Palais, Paris) combine des figures et des paysages de deux différents tableaux. Les gravures de Claude, comme on le voit dans cette exposition, réfèrent souvent à des sujets parallèles, traités comme dessins, tableaux ou autres gravures. Ces croisements de media et de sujets rendent souvent parfois la datation des gravures. Claude a gravé quarante-quatre différents sujets, dont seulement douze référant à des tableaux connus et cinq présentant des similitudes avec certains tableaux. C'est difficile d'établir si chacune de ces dix-sept gravures a été crée avant ou après les tableaux. En tout cas, dans cette exposition on constate que la majorité des gravures de Claude sont des créations indépendantes, non liées directement à des œuvres dans des autres media.

Il serait rare de trouver même une seule planche de la suite des Feux d'artifices de Claude. Dans l'exposition à Washington DC et à Paris en 1982-1983, il y avait treize gravures des Feux. Maintenant à Oxford et Francfort, il y a seize différentes gravures de cette suite. Pouvoir contempler des tels ensembles est extraordinaire. Dans son texte, Sonnabend présente des descriptions très détaillées, terminant avec l'idée que les Feux d'artifices en tant que suite « se développent comme un film ».

Claude, comme Rembrandt, était un graveur expérimental. Dans cette exposition, par exemple, il y a une magnifique épreuve de Berger et Bergère conversant, 1651 (premier de sept états, British Museum). 


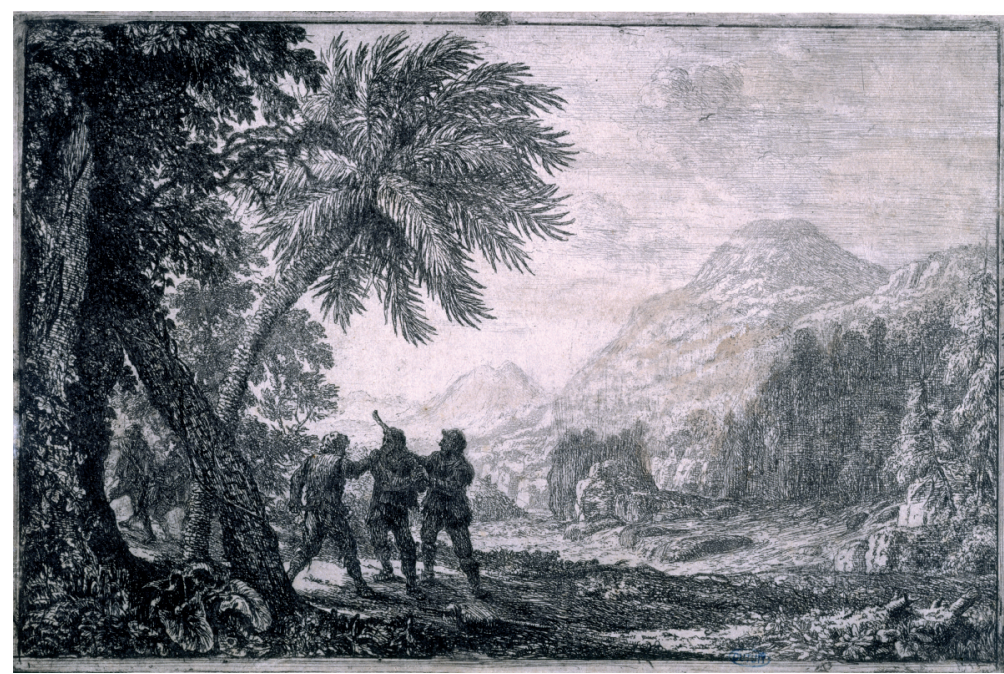

Cat. Nr. 90 Scène de Brigands ( $1^{\text {er }}$ état de 9 états, avec lavis ajouté), 1635. Musée du Petit Palais, Paris.

Cat. Nr. 101 La Tour carrée ( 2 état de 3 états), 1637. BnF, Estampes, Paris.

Lépreuve en question a été retouchée à l'aquarelle par Claude, envisageant les changements qu'il pensait faire dans les prochains états. Cette gravure a commencé comme une reproduction d'un tableau, mais est devenue une œuvre très différente. Dans ce sens, on pourrait référer aussi à Mercure et Argus (premier de trois états, musée d'art et d'histoire, Genève, reproduit en catalogue, mais non exposé), sous forme d'une contre-épreuve, avec aquarelle ajoutée, indiquant des changements à considérer pour les prochains états. En terminant les textes du catalogue, Christian Rümelin note que cette exposition cherche à mettre les gravures de Claude dans un contexte plus large en les comparant surtout avec celles de Rembrandt (16061669). Rembrandt, se trouvant dans le même contexte historique que Claude, a subi les mêmes influences, influences incluant certainement des gravures de l'époque d'Hendrik Goudt et d'Hercules Segers. L'en-

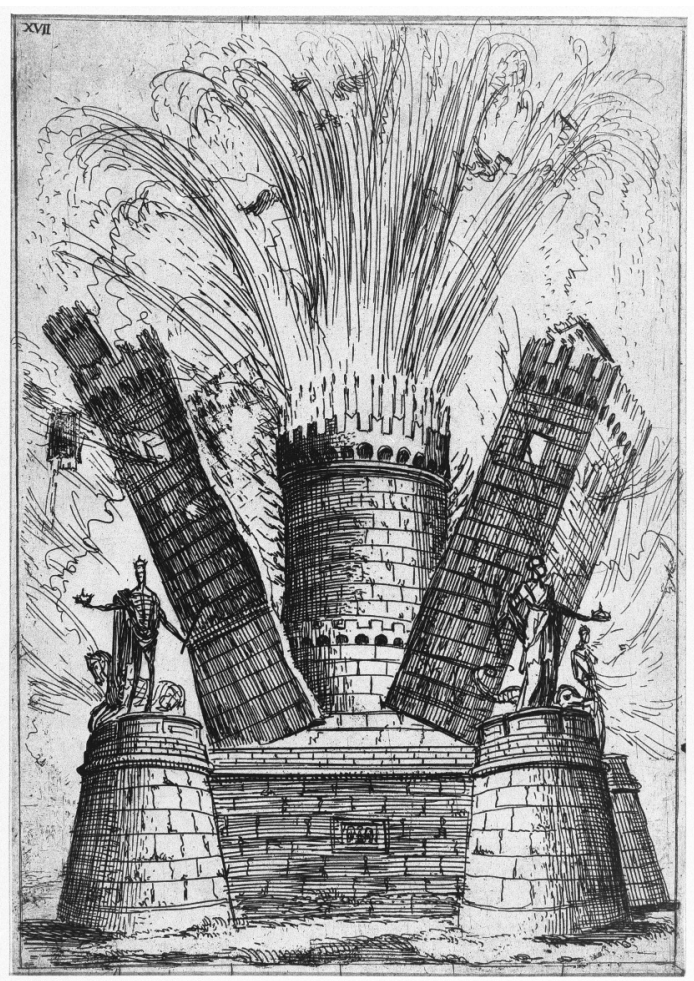
semble de ces influences a changé dramatiquement la conception du paysage et de la continuité spatiale dans l'art de Claude.

Pour les auteurs du catalogue de cette exposition, Claude est un graveur qui n'essaie pas de simplement traduire ou d'exprimer des idées originales. Au contraire, il se sert de la gravure afin de développer des idées, qui éventuellement se trouveront dans ses dessins et tableaux ou dans d'autres gravures. Les changements dans le développement d'une gravure constituent une partie d'un procédé et ainsi conduisent à différentes impressions et différentes directions. Ceci ne pourrait pas s'appliquer autant aux dessins ou aux tableaux (sauf pour des dessins ou tableaux « en série », c'est-à-dire les multiples dessins des détails de la SainteAnne de Léonard, récemment exposés au Louvre à Paris), parce que ces deux media n'offrent pas un procédé comparable aux possibilités de 
la gravure. Un artiste est très limité en essayant de corriger ou de développer un dessin, une aquarelle ou un pastel. En revanche, avec la gravure, Claude et aussi Rembrandt pouvaient faire un certain nombre de changements en créant d'autres états.

L'importance des 《états » dans l'histoire de la gravure, et la recon-

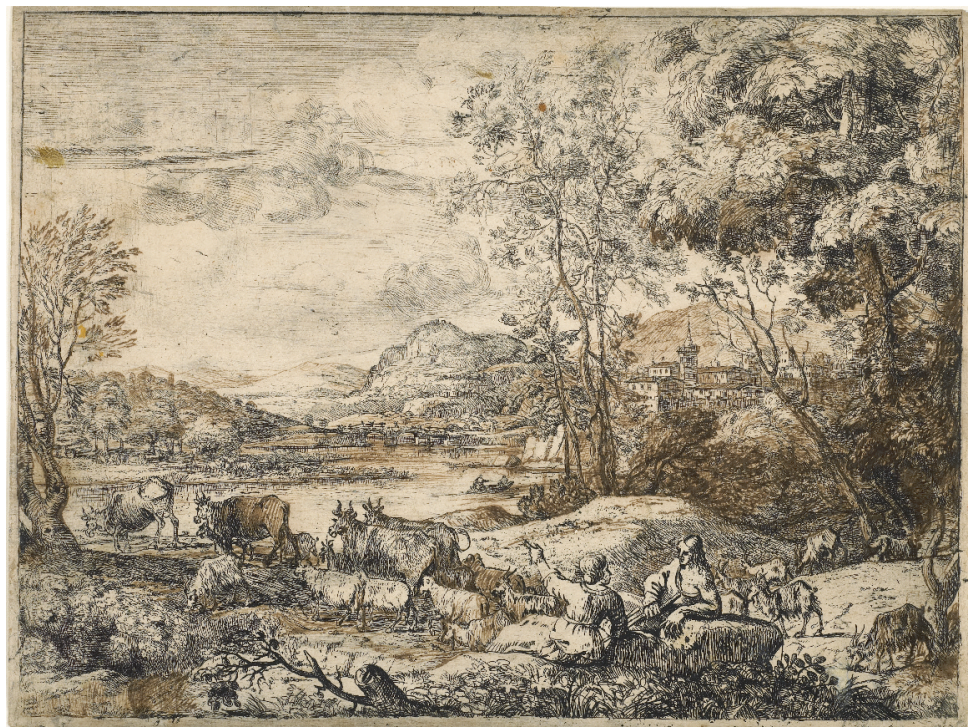
naissance de cette importance de la part du public, a vraiment commencé au moment de l'époque de Claude et de Rembrandt. Cette évolution s'est épanouie au début du XIX siècle avec les gravures de Goya, Géricault et Delacroix, et est arrivée à une conclusion au temps des impressionnistes. Par exemple, en créant vers 1880 la publication Le Jour et la Nuit (qui n’a jamais été publiée), Cassatt, Degas et Pissarro, comme bien d'autres graveurs de leur temps, ont trouvé qu'il y avait des possibilités d'expression artistique en gravure qui n'existent pas en peinture ou en dessin. En effet, chaque touche d'un pinceau mise sur un tableau détruit pour toujours les impressions peintes antérieurement. Le tableau final, une accumulation « d'états », représente ainsi la destruction de toutes les précédentes « impressions ». Alors que, les impressions de chacun des « états » des gravures de Claude peuvent être conservées et considérées comme des œuvres d'art autonomes.

La présentation de ces cent trente et une œuvres de Claude à Oxford et à Francfort, ensemble avec les recherches du catalogue descriptif, représente une contribution importante dans le domaine de l'histoire des arts graphiques du XVII siècle. Cette exposition éta-

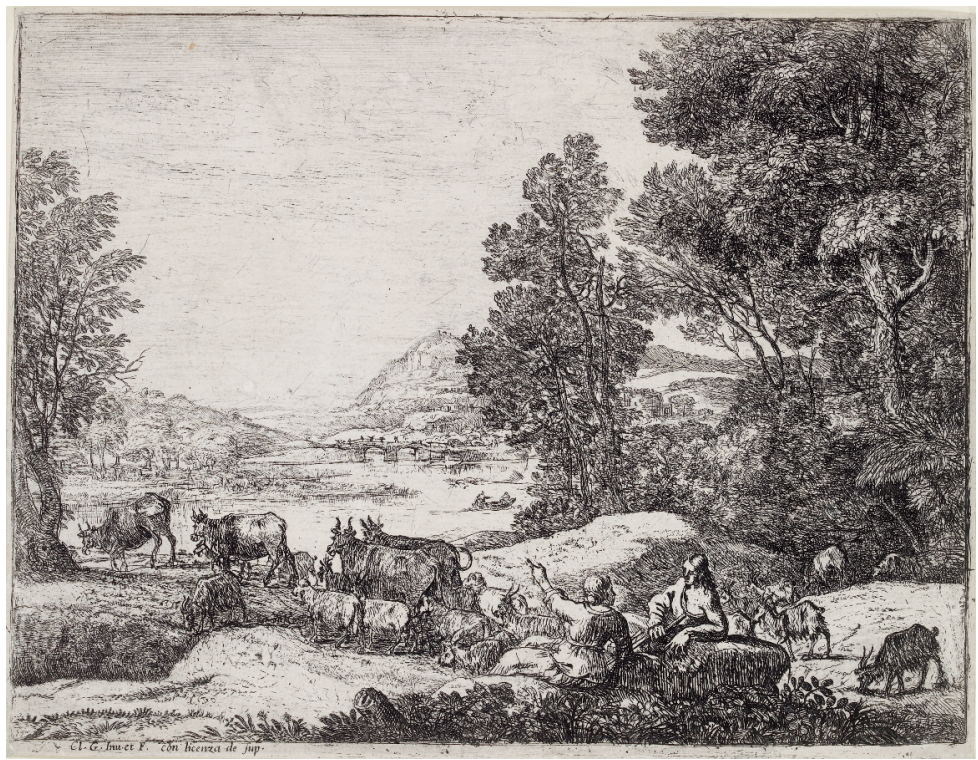
blit également une nouvelle base pour des études futures sur l'œuvre de Claude Gellée Le Lorrain.

Cat. Nr. 125 Berger et Bergère conversant ( $1^{\text {er }}$ état de 7 états, avec aquarelle ajoutée), 1651 British Museum, Londres.

Cat. Nr. 127 Berger et Bergère conversant ( $5^{\mathrm{e}}$ état de 7 états), 1651. Ashmolean, Oxford. 